\title{
Treatment of Adult Eosinophilic Esophagitis with Diet
}

\author{
Alfredo J. Lucendo ${ }^{a}$ Angel Arias ${ }^{b}$ \\ ${ }^{a}$ Department of Gastroenterology, Hospital General de Tomelloso, Tomelloso, and ${ }^{b}$ Research Support Unit, \\ Hospital General La Mancha Centro, Alcázar de San Juan, Spain
}

\begin{abstract}
Key Words
Eosinophilic esophagitis - Dietary therapy · Diet · Adult · Allergy testing
\end{abstract}

\begin{abstract}
Background/Aims: A growing body of research indicates that dietary therapy is an effective treatment option for children with eosinophilic esophagitis (EoE). Its ability to achieve sustained symptomatic and histological responses is comparable to that of topical steroids, but avoids their use and side effects. Recent studies also support dietary therapy as a preferred treatment option for adult patients. Methods: A comprehensive literature review was carried out to assess the efficacy of the most commonly used dietary interventions in inducing and maintaining disease remission in adult EoE patients as compared with results from pediatric studies. Results: The only available study assessing exclusive feeding with an elemental diet carried out in adults demonstrated a 94\% disease remission rate, comparable to the high efficacy reported in pediatric studies. The major drawbacks of this therapy are poor adherence and a rapid disease recurrence after resumption of a normal diet. Although there is limited data on skin allergy testing-directed food elimination in adult patients, the highest efficacy rates observed do not exceed $26 \%$ histological remission. Prospective studies on empirical six-food elimination diets in adults provided efficacy rates comparable to those reported in children, achieving disease remission in approximately $75 \%$ of patients. How-
\end{abstract}

ever, specific food triggers can only be identified after documented recurrence of eosinophilic inflammation as determined with repeated endoscopic biopsies. Prolonged avoidance of the offending food(s) produces sustained remission of adult EoE. Conclusions: Diet is a feasible therapeutic option for adult EoE patients, providing them with the same effective drug-free alternative as in pediatric patients.

(c) 2014 S. Karger AG, Basel

\section{Introduction}

Eosinophilic esophagitis (EoE), an emerging esophageal disorder characterized by marked tissue eosinophilia and symptoms of esophageal dysfunction, usually persists from childhood to adulthood $[1,2]$. Although the disease was first described 20 years ago in adults $[3,4]$, most of the available research has been conducted on pediatric patients.

Since its initial descriptions, EoE has been associated with food allergies, with patients usually presenting various atopic symptoms along with sensitizations to food allergens, which have been documented in a high proportion of patients of all ages [5]. However, the high response rate to food elimination diets, especially amino acidbased elemental diets [6-8] and empirical six-food elimination diets (SFEDs) $[9,10]$, indicates that allergic sensitization to commonly consumed foods is involved in the pathogenesis of the disease. Indeed, sequential food rein-

\section{KARGER}

E-Mail karger@karger.com

www.karger.com/ddi
(C) 2014 S. Karger AG, Basel

0257-2753/14/0322-0120\$39.50/0
Alfredo J. Lucendo, MD, PhD, FEBGH

Department of Gastroenterology, Hospital General de Tomelloso

Vereda de Socuéllamos, s/n

ES-13700 Tomelloso (Spain)

E-Mail alucendo@vodafone.es 
troduction with documented disease recurrence is the most accurate way to identify EoE food triggers in both children and adult patients [11-13].

The management of EoE in clinical practice remains somewhat controversial and eclectic, with treatment modalities including the use of swallowed steroids with a topical effect, various dietary modifications, endoscopic dilation, and even administration of several monoclonal antibodies [14]. As of yet, a commonly accepted algorithm for treating patients is lacking, mostly due to limited information about the long-term efficacy of the different therapies in modifying the natural history of the disease and the associated subepithelial fibrosis [14-16]. As a result, a wide variability, both in the standard of care given to EoE patients and in the adherence to proposed international guidelines, has been documented in clinical practice $[17,18]$.

There has been a renewal of interest in dietary treatment of EoE, with an increasing number of studies demonstrating that dietary therapy can be an effective treatment option for pediatric patients, in whom it produces a drug-free symptomatic and histological remission. Recently published prospective studies have also noted symptomatic and histological remission in adult patients after following an elimination diet. This article aims to review and critically discuss the available evidence in order to assess the efficacy of treating adult EoE patients through dietary intervention.

\section{Rationale for Dietary Treatment in Adult EoE Patients}

Differences in clinical presentation, endoscopic aspects, and response to treatment have all been reported for EoE patients of different ages, giving rise to the question of whether adult and pediatric EoE are manifestations of a single entity or are, in fact, two distinct disorders. Most studies point to a common pathogenesis and similar histopathological features in both children and adults, positing that differences in symptoms between the two age groups can be explained by a patient's ability to communicate esophageal dysfunction $[2,19,20]$. Although no specific studies have been published to date, differences in endoscopic features suggest that signs of active acute inflammation, such as edema and exudates, predominate in children while manifestations of chronic inflammation, such as rigid rings and strictures, occur more frequently in adults. These differences have been related to fibrous remodeling, a phenomenon that has been reported in both age groups [21, 22], but which seems to be reversible in children $[15,23]$ while having a tendency to persist in adults [16]. In addition, no differences between pediatric and adult EoE patients have been noted regarding patient response to currently used pharmacological modalities; moreover, allergic sensitization patterns are similar across age groups. As a result, because EoE is currently considered to be a single disease across all age groups, the expected efficacy of dietary intervention in adults should reproduce the results observed in children.

\section{Dietary Interventions for Adult EoE Patients}

The three major types of dietary interventions assessed in children with EoE have also been evaluated in prospective studies conducted with adult patients over the past few years. They include (1) an elemental formula diet with complete elimination of all table foods, (2) a skin allergy testing-directed elimination diet, and (3) an empirical elimination diet based on common food allergens.

\section{Elemental Diet in Adult EoE}

The first evidence of the efficacy of dietary intervention against EoE was reported in 1995 by Kelly et al. [6], who used an amino acid-based elemental formula exclusively for feeding 10 children with intense esophageal eosinophilia attributed to refractory gastroesophageal reflux. Eight patients exhibited complete resolution of the disease while 2 others presented symptomatic improvement, all in parallel with an average reduction of peak eosinophil counts from 41 eosinophils/high-power field (HPF) to 0.5 eosinophil/HPF after 8 weeks of treatment. Subsequent studies and case reports have repeatedly confirmed these results in pediatric and adolescent patients [7-9, 24-28]. Despite the fact that no randomized controlled trials are available, firm evidence indicates that elemental diets in pediatric EoE have an overall effectiveness of around $90 \%$ in reducing peak eosinophil counts to below 15 eosinophils/HPF.

The positive, extensively documented results obtained in children were not corroborated in adults until 2013, when Peterson et al. [29] prospectively recruited 29 adult EoE patients to follow an elemental diet for a 4-week period. Among the 18 patients who completed the study protocol, 13 achieved a complete histological response (defined as $<10$ eosinophils/HPF), with 4 additional patients presenting peak eosinophil reductions of over $50 \%$ with regard to the baseline. Analysis per protocol showed 
an overall efficacy of $94.4 \%$ in terms of histological remission, which fell to $58.6 \%$ when analyzed as intention to treat. Difficulties in adhering to the diet caused 3 enrolled patients to abandon the study on the first day, while 8 others consumed solid food during the initial 2 weeks. Moreover, EoE recurred in every patient within 2-7 days after normal food reintroduction, which brings into question the feasibility of exclusive feeding with elemental formulas in the practical management of EoE, especially in the absence of a controlled food reintroduction protocol.

Despite the fact that all of the available results to date indicate that elemental diets are as efficient as topical steroids in inducing EoE remission, the absence of drugrelated side effects may not make up for all of the drawbacks of this type of dietary intervention. In addition to the psychological and social effects of complete avoidance of table foods, the unsavory taste of amino acid-based elemental formulas $(80 \%$ of pediatric patients must be fed through a nasogastric tube in some series [8]), their high cost, and their impracticality for chronic use make this type of dietary intervention an unfeasible treatment option for older children and adults.

\section{Skin Allergy Testing-Directed Elimination Diet}

The impracticality of elemental diets for long-term use prompted investigators to try to identify and remove specific food allergens from the diet using skin allergy testing results as a guide. It was hoped that this attempt to obtain comparable results with less drastic measures would allow patients to follow a near-normal diet, thus avoiding deterioration in their overall health and quality of life.

In 2002, the first approach to a directed food exclusion treatment for pediatric EoE patients was developed by Spergel et al. [30], who identified potential food triggers for EoE by using a combination of skin prick tests (SPTs) and atopy patch tests (APTs). After advising patients to avoid consumption of those foods showing a positive result, histopathological remission was achieved in 18 out of the 24 children recruited (77\%). The overall efficacy rates of skin allergy testing-directed diets in pediatric EoE patients have been subsequently updated by the same research group $[25,31,32]$, with their last paper retrospectively documenting complete disease resolution in $53 \%$ of patients following an allergy testing (SPT and APT)-directed exclusion diet.

Unfortunately, the $77 \%$ efficacy rate observed in the initial study [31] has never been replicated [10, 24, 33]. In fact, in several subsequent studies, no patients managed to achieve a complete histological response $[28,34]$. Con- troversy thus exists as to the actual efficacy of skin allergy testing-directed exclusion diets, especially with regard to the methodology for carrying out and reading APT results.

As with elemental diets, studies carried out in adults on the utility of skin allergy testing-directed elimination diets are also scarce, with the best documented research to date being that conducted by Molina-Infante et al. [35]. These authors excluded every food which gave a positive result ina SPT, prick-prick test, or APT, documenting disease remission in only 4 of the 15 recruited patients (26\%). The second study conducted with adults documented symptomatic improvement in only 1 of the $6 \mathrm{EoE}$ patients studied, with no signs of histological remission [36].

Possible explanations for the wide variability in efficacy rates of skin allergy testing-directed food elimination include increasing evidence of a cell-mediated delayed hypersensitivity reaction rather than a predominantly IgE-mediated response as the main pathophysiological mechanism leading to EoE [37]. In fact, foodspecific IgE serum measurements and SPTs have proven to be neither sensitive nor specific methods for retrospectively predicting EoE triggers in adult patients [12,13]. In addition, the immaturity of the gastrointestinal enzymatic system and the mucosal barrier in small children favors the absorption of partially digested peptides from the diet. This causes a primed immune response through Th2 lymphocyte stimulation, cytokine secretion, and IgE production [38]. Because this process diminishes with age, adult patients may present reduced rates of IgE hypersensitivity to food.

\section{Empirical Elimination Diets Based on Common Food} Allergens as a Treatment for Adult EoE

In 2006, in an attempt to allow patients solid food consumption to overcome the significant drawbacks of elemental diets, Kagalwalla et al. [9] explored a dietary alternative consisting of the elimination of several, but not all, intact food proteins from the diet of pediatric EoE patients, putting them on an SFED. Foods most commonly associated with food allergies in children were excluded, including milk protein, soy, eggs, wheat, peanuts/tree nuts, and seafood. After a 6-week period, 26 of the 35 children treated $(74 \%)$ achieved complete histological remission $(<10$ eosinophils/HPF), with a partial remission (1120 eosinophils/HPF) documented in 3 others. A subsequent retrospective study also developed in children extended the 'classic' SFED to include foods with a positive result in allergy tests [10], achieving histological remission in $81 \%$ of the recruited patients. 
Results for adult patients come from two prospective studies published within the last year. Gonsalves et al. [12] recruited 50 adult EoE patients from the same geographical area studied in Kagalwalla's original report. After following an SFED extended to include foods giving a positive skin allergy test result, $70 \%$ of the study subjects showed complete disease remission $(<10$ eosinophils/ HPF). Lucendo et al. [13] carried out the only available study of adult EoE patients in Europe using an empirical SFED approach. Histological remission $(<15$ eosinophils/ HPF) was documented in $73 \%$ of the study subjects, with significant improvement of symptoms observed in every responder patient. It is worth noting that in the latter study, the 'classic' SFED was extended to legumes, rice, and corn since these foods are common allergens in the study population.

Although limited in number, studies assessing empirical SFEDs exhibit a fairly uniform efficacy rate, successfully inducing disease remission in 3 out of $4 \mathrm{EoE}$ patients, regardless of age. This dietary intervention offers the advantage of improved compliance in comparison to elemental diets. In addition, it can be implemented without performing skin allergy tests, making the elimination of common food allergens a feasible initial approach for the therapeutic management of EoE patients of all ages.

\section{Identification of Food Triggers for EoE}

According to the latest international consensus guidelines for managing EoE [5], food triggers can currently only be identified by documenting EoE recrudescence upon specific food reintroduction after disease remission has been achieved through specific food antigen avoidance. In this context, positive SPT, serum IgE, and APT results should not be used alone to diagnose food triggers in EoE patients. Likewise, food restrictions should not be recommended if based exclusively on a positive allergy test result. The recommended strategy of sequential food reintroduction ('food challenge') under endoscopic and bioptic monitoring has been used by several authors to identify EoE triggers after achieving disease remission in both children [11,25] and adults [12,13]. Identified food triggers are similar across the various studies, with cow's milk and wheat being the major food triggers for EoE in around $50 \%$ of pediatric and adult cases, regardless of geographical area. Differences in the prevalence of other food triggers have been documented between American and European studies, raising the question of whether empirical elimination diets should be tailored to the staple diet and food allergy sensitization patterns of patients from specific geographical areas.

\section{Sustained Efficacy of Dietary Treatment}

International consensus guidelines recommend longterm exclusion of those foods identified as being responsible for EoE from the diet [5]. Information on the possibility of inducing tolerance to food allergens triggering EoE in children after prolonged exclusion is scarce and disappointing since the disease universally recurs upon food reintroduction, even after a remission period of up to 4 years [11].

Two studies have provided data on the long-term efficacy of eliminating from the diet specifically identified food triggers for adult EoE. In the first one (only published as an abstract), 9 patients demonstrated no disease recurrence after 1 year of dietary maintenance [39]. In the second study, the authors found that disease remission was maintained in all of the 25 patients adhering to the diet for up to a 3-year period [13].

\section{Emerging Dietary Schemes for the Near Future}

After identifying the major food triggers for EoE by having patients follow empirical SFEDs and then carrying out sequential food challenge, simplified four-food elimination diets (FFEDs) have emerged as an easier alternative which might improve patient acceptance of dietary restrictions, especially since it also reduces the number of endoscopic exams and overall time needed to complete the food reintroduction protocol. The first group to study the efficacy of FFEDs was Gonsalves et al. [40], who had 13 adult patients follow a milk-, wheat-, egg-, and soy-free diet. Sixty percent of the study subjects achieved histological remission $(<10$ eosinophils/HPF) after a 6-week period. An optimized FFED, which excluded milk, gluten, eggs, and legumes (including soy and peanuts), was later assessed prospectively in a multicenter study carried on 35 adult EoE patients in Spain [Molina-Infante et al., poster presentation at Falk symposium 189]. In this study, $57 \%$ of patients achieved histological remission $(<10$ eosinophils/HPF) after following an FFED, with nonresponders given the option of following an SFED as a rescue therapy. By combining these two strategies, an overall remission rate of $88 \%$ was achieved. 


\section{Conclusion}

Dietary therapy constitutes a feasible and effective treatment option for adult EoE patients, with disease remission rates as high as those previously demonstrated in pediatric patients. Our review of the literature found that the high efficacy of elemental diets is undermined by low adherence rates and other practical disadvantages, and that the limited number of studies assessing skin allergy testing-directed food elimination show unsatisfactory re- sults. We thus feel that, according to the available research and results, empirical SFEDs are the initial dietary intervention of choice for adult EoE patients. Preliminary data on FFEDs indicate that this may be an additional option to consider in the near future.

\section{Disclosure Statement}

The authors declare no conflicts of interest.

\section{References}

$>_{1}$ DeBrosse CW, Franciosi JP, King EC, Butz BK, Greenberg AB, Collins MH, Abonia JP, Assa'ad A, Putnam PE, Rothenberg ME Long-term outcomes in pediatric-onset esophageal eosinophilia. J Allergy Clin Immunol 2011;128:132-138.

2 Lucendo AJ, Sánchez-Cazalilla M: Adult versus pediatric eosinophilic esophagitis: important differences and similarities for the clinician to understand. Expert Rev Clin Immunol 2012;8:733-745.

-3 Attwood SE, Smyrk TC, Demeester TR, Jones JB: Esophageal eosinophilia with dysphagia. A distinct clinicopathologic syndrome. Dig Dis Sci 1993;38:109-116.

-4 Straumann A, Spichtin HP, Bernoulli R, Loosli J, Vogtlin J: Idiopathic eosinophilic esophagitis: a frequently overlooked disease with typical clinical aspects and discrete endoscopic findings. Schweiz Med Wochenschr 1994;124:1419-1429.

-5 Liacouras CA, Furuta GT, Hirano I, Atkins D, Attwood SE, Bonis PA, Burks AW, Chehade M, Collins MH, Dellon ES, Dohil R, Falk GW, Gonsalves N, Gupta SK, Katzka DA, Lucendo AJ, Markowitz JE, Noel RJ, Odze RD, Putnam PE, Richter JE, Romero Y, Ruchelli E, Sampson HA, Schoepfer A, Shaheen NJ, Sicherer SH, Spechler S, Spergel JM, Straumann A, Wershil BK, Rothenberg ME, Aceves SS: Eosinophilic esophagitis: updated consensus recommendations for children and adults. J Allergy Clin Immunol 2011;128:3-20.

6 Kelly KJ, Lazenby AJ, Rowe PC, Yardley JH, Perman JA, Sampson HA: Eosinophilic esophagitis attributed to gastroesophageal reflux: improvement with an amino acid-based formula. Gastroenterology 1995;109:15031512.

7 Markowitz JE, Spergel JM, Ruchelli E, Liacouras CA: Elemental diet is an effective treatment for eosinophilic esophagitis in children and adolescents. Am J Gastroenterol 2003;98: 777-782.
Liacouras CA, Spergel JM, Ruchelli E, Verma R, Mascarenhas M, Semeao E, Flick J, Kelly J, Brown-Whitehorn T, Mamula P, Markowitz JE: Eosinophilic esophagitis: a 10-year experience in 381 children. Clin Gastroenterol Hepatol 2005;3:1198-1206.

$\checkmark 9$ Kagalwalla AF, Sentongo TA, Ritz S, Hess T, Nelson SP, Emerick KM, Melin-Aldana H, Li BU: Effect of six-food elimination diet on clinical and histologic outcomes in eosinophilic esophagitis. Clin Gastroenterol Hepatol 2006;4:1097-1102.

10 Henderson CJ, Abonia JP, King EC, Putnam PE, Collins MH, Franciosi JP, Rothenberg ME: Comparative dietary therapy effectiveness in remission of pediatric eosinophilic esophagitis. J Allergy Clin Immunol 2012; 129:1570-1578

11 Kagalwalla AF, Shah A, Li BU, Sentongo TA, Ritz S, Manuel-Rubio M, Jacques K, Wang D, Melin-Aldana H, Nelson SP: Identification of specific foods responsible for inflammation in children with eosinophilic esophagitis successfully treated with empiric elimination diet. J Pediatr Gastroenterol Nutr 2011;53: 145-149.

12 Gonsalves N, Yang GY, Doerfler B, Ritz S, Ditto AM, Hirano I: Elimination diet effectively treats eosinophilic esophagitis in adults; food reintroduction identifies causative factors. Gastroenterology 2012;142:1451-1459.

13 Lucendo AJ, Arias A, Gonzalez-Cervera J, Yague-Compadre JL, Guagnozzi D, Angueira T, Jimenez-Contreras S, Gonzalez-Castillo S, Rodriguez-Domingez B, De Rezende LC, Tenias JM: Empiric 6-food elimination diet induced and maintained prolonged remission in patients with adult eosinophilic esophagitis: a prospective study on the food cause of the disease. J Allergy Clin Immunol 2013;131: 797-804.

14 Gonzalez-Castillo S, Arias A, Lucendo AJ: Treatment of eosinophilic esophagitis: how should we manage the disease? J Clin Gastroenterol 2010;44:663-671.
15 Lieberman JA, Morotti RA, Konstantinou GN, Yershov O, Chehade M: Dietary therapy can reverse esophageal subepithelial fibrosis in patients with eosinophilic esophagitis: a historical cohort. Allergy 2012;67:1299-1307.

-16 Lucendo AJ, Arias A, De Rezende LC, YagueCompadre JL, Mota-Huertas T, GonzalezCastillo S, Cuesta RA, Tenias JM, Bellon T: Subepithelial collagen deposition, profibrogenic cytokine gene expression, and changes after prolonged fluticasone propionate treatment in adult eosinophilic esophagitis: a prospective study. J Allergy Clin Immunol 2011; 128:1037-1046.

17 Spergel JM, Book WM, Mays E, Song L, Shah SS, Talley NJ, Bonis PA: Variation in prevalence, diagnostic criteria, and initial management options for eosinophilic gastrointestinal diseases in the United States. J Pediatr Gastroenterol Nutr 2011;52:300-306.

18 Lucendo AJ, Arias A, Molina-Infante J, Rodriguez-Sanchez J, Rodrigo L, Nantes O, Perez-Arellano E, de la RS, Perez-Aisa A, Barrio $\mathrm{J}$ : Diagnostic and therapeutic management of eosinophilic oesophagitis in children and adults: results from a Spanish registry of clinical practice. Dig Liver Dis 2013;45:562-568.

19 Straumann A, Aceves SS, Blanchard C, Collins MH, Furuta GT, Hirano I, Schoepfer AM, Simon D, Simon HU: Pediatric and adult eosinophilic esophagitis: similarities and differences. Allergy 2012;67:477-490.

20 Putnam PE: Eosinophilic esophagitis in children: clinical manifestations. Gastrointest Endosc Clin N Am 2008;18:11-23.

21 Chehade M, Sampson HA, Morotti RA, Magid MS: Esophageal subepithelial fibrosis in children with eosinophilic esophagitis. J Pediatr Gastroenterol Nutr 2007;45:319-328.

22 Aceves SS, Newbury RO, Dohil R, Bastian JF, Broide DH: Esophageal remodeling in pediatric eosinophilic esophagitis. J Allergy Clin Immunol 2007;119:206-212. 
23 Aceves SS, Newbury RO, Chen D, Mueller J, Dohil R, Hoffman H, Bastian JF, Broide DH: Resolution of remodeling in eosinophilic esophagitis correlates with epithelial response to topical corticosteroids. Allergy 2010;65: 109-116.

-24 Kagalwalla AF, Amsden K, Shah A, Ritz S, Manuel-Rubio M, Dunne K, Nelson SP, Wershil BK, Melin-Aldana H: Cow's milk elimination: a novel dietary approach to treat eosinophilic esophagitis. J Pediatr Gastroenterol Nutr 2012;55:711-716.

25 Spergel JM, Brown-Whitehorn TF, Cianferoni A, Shuker M, Wang ML, Verma R, Liacouras CA: Identification of causative foods in children with eosinophilic esophagitis treated with an elimination diet. J Allergy Clin Immunol 2012;130:461-467.

-26 Ferreira CT, Vieira MC, Vieira SM, Silva GS, Yamamoto DR, Silveira TR: Eosinophilic esophagitis in 29 pediatric patients (in Portuguese). Arq Gastroenterol 2008;45:141-146.

27 De Agustin JC, Sanz N, Canals MJ, Alvarez E, Morales JL, Soler J, Ollero JC, Vazquez J: Successful medical treatment of two patients with eosinophilic oesophagitis. J Pediatr Surg 2002;37:207-213.

28 Al-Hussaini A, Al-Idressi E, Al-Zahrani M: The role of allergy evaluation in children with eosinophilic esophagitis. J Gastroenterol 2013;48:1205-1212.
29 Peterson KA, Byrne KR, Vinson LA, Ying J, Boynton KK, Fang JC, Gleich GJ, Adler DG, Clayton F: Elemental diet induces histologic response in adult eosinophilic esophagitis. Am J Gastroenterol 2013;108:759-766.

30 Spergel JM, Beausoleil JL, Mascarenhas M, Liacouras CA: The use of skin prick tests and patch tests to identify causative foods in eosinophilic esophagitis. J Allergy Clin Immunol 2002;109:363-368.

- 31 Spergel JM, Brown-Whitehorn T, Beausoleil JL, Shuker M, Liacouras CA: Predictive values for skin prick test and atopy patch test for eosinophilic esophagitis. J Allergy Clin Immunol 2007;119:509-511.

32 Spergel JM, Brown-Whitehorn TF, Beausoleil JL, Franciosi J, Shuker M, Verma R, Liacouras CA: 14 years of eosinophilic esophagitis: clinical features and prognosis. J Pediatr Gastroenterol Nutr 2009;48:30-36.

33 Liacouras CA, Spergel JM, Ruchelli E, Verma R, Mascarenhas M, Semeao E, Flick J, Kelly J, Brown-Whitehorn T, Mamula P, Markowitz JE: Eosinophilic esophagitis: a 10-year experience in 381 children. Clin Gastroenterol Hepatol 2005;3:1198-1206.

34 Rizo Pascual JM, De La Hoz CB, Redondo VC, Terrados CS, Roy AG, Riesco Lopez JM, Camarero SC: Allergy assessment in children with eosinophilic esophagitis. J Investig Allergol Clin Immunol 2011;21:59-65.
5 Molina-Infante J, Martin-Noguerol E, Alvarado-Arenas $\mathrm{M}$, Porcel-Carreno SL, Jimenez-Timon S, Hernandez-Arbeiza FJ: Selective elimination diet based on skin testing has suboptimal efficacy for adult eosinophilic esophagitis. J Allergy Clin Immunol 2012; 130:1200-1202.

36 Simon D, Straumann A, Wenk A, Spichtin H, Simon HU, Braathen LR: Eosinophilic esophagitis in adults - no clinical relevance of wheat and rye sensitizations. Allergy 2006;61:14801483.

37 Lucendo AJ, Lucendo B: An update on the immunopathogenesis of eosinophilic esophagitis. Expert Rev Gastroenterol Hepatol 2010;4: 141-148.

38 Bernardo D: Human intestinal dendritic cells as controllers of mucosal immunity. Rev Esp Enferm Dig 2013;105:279-290.

39 Gonsalves N, Doerfler B, Hirano I: Long term maintenance therapy with dietary restriction in adults with eosinophilic esophagitis (abstract). Gastroenterology 2011;140(5 suppl 1):S180-S181.

40 Gonsalves N, Doerfler B, Schwartz S, Yang G-Y, Zalewsky A, Amsden K, Mughal S, Manuel-Rubio M, Melin-Aldana $\mathrm{H}$, Wershil BK, Hirano I, Kagalwalla AF: Prospective trial of four food elimination diet demonstrates comparable effectiveness in the treatment of adult and pediatric eosinophilic esophagitis. Gastroenterology 2013;144(suppl 1):S154. 